NEUROSCIENCE

\section{The Speaking Brain}

Peter Hagoort ${ }^{1,2}$ and Willem J. M. Levelt ${ }^{1}$

$\mathrm{H}$ ow does intention to speak become the action of speaking? It involves the generation of a preverbal message that is tailored to the requirements of a particular language, and through a series of steps, the message is transformed into a linear sequence of speech sounds $(1,2)$. These steps include retrieving different kinds of information from memory (semantic, syntactic, and phonological), and combining them into larger structures, a process called unification. Despite general agreement about the steps that connect intention to articulation, there is no consensus about their temporal profile or the role of feedback from later steps $(3,4)$. In addition, since the discovery by the French physician Pierre Paul Broca (in 1865) of the role of the left inferior frontal cortex in speaking, relatively little progress has been made in understanding the neural infrastructure that supports speech production (5). One reason is that

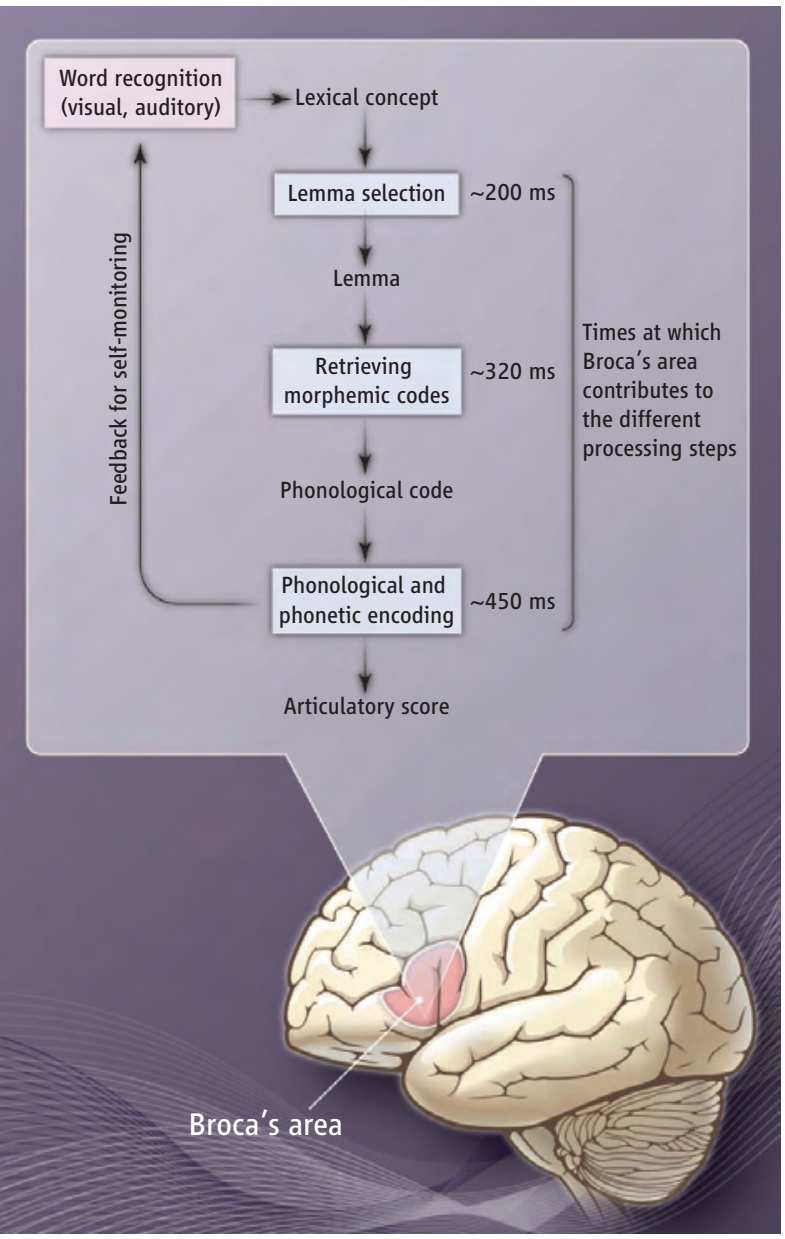
the characteristics of natural language are uniquely human, and thus the neurobiology of language lacks an adequate animal model. But on page 445 of this issue, Sahin et al. (6) demonstrate, by recording neuronal activity in the human brain, that different kinds of linguistic information are indeed sequentially processed within Broca's area.

Sahin et al. had the unique opportunity to record from three patients with epilepsy during presurgical preparation. Depth electrodes were implanted in Broca's area and the anterior temporal cortex, and local field potentials were recorded while the patients were engaged in a language production task. The subjects were asked either to read silently words presented on a screen, or to silently produce the inflected form of the presented

${ }^{1}$ Max Planck Institute for Psycholinguistics, NL-6500 HB Nijmegen, Netherlands. 2Donders Institute for Brain, Cognition and Behaviour, Radboud University Nijmegen, Netherlands. E-mail: peter.hagoort@donders.ru.nl
Recordings of electrical activity in the human brain reveal the fine-tuned, stepwise neuronal processing of language and speech.

From intention to articulation. Shown is an adapted version of the lexical encoding model for speech production (2), specifying steps in the paradigm used by Sahin et al. Based on the visual input, a lemma is selected that specifies the syntactic features of a lexical concept. For instance, for the lemma horse, it specifies that it is a count noun. In addition, the morphemic codes are retrieved. For instance, when the speaker wants to produce the plural form of horse, the codes for both the stem and the plural suffix are retrieved. Next, the phonological codes for each morpheme are retrieved, combined, and transformed into a motor command to the articulatory system. The approximate times (in milliseconds) at which Broca's area contributes to the different processing steps are shown. The late (i.e., at 500 to $600 \mathrm{~ms}$ ) monophasic component observed in the temporal lobe (6) might reflect self-monitoring of the speech output.

were not only temporally separated, but also spatially segregated at a scale of only a few millimeters, which is below the effective spatial resolution of standard functional magnetic resonance imaging of brain activity.

These data are relevant for both cognitive models of speech production and for accounts on the role of Broca's area. The time course is clear evidence for the sequentiality of different access and unification operations in speaking, and is consistent with the few estimates in the literature $(7,8)$. Moreover, both the anatomical and the temporal segregation of word-encoding operations in Broca's area are in line with the view that this region is involved with each of these encoding operations and their unification over time. Feedback operations among these processes cannot be excluded. However, the fine-grained temporal and spatial separation of these steps suggests that we are witnessing the "first go" process at work here.

Both functional magnetic resonance imaging and lesion studies have shown that Broca's area is also involved in processing inflectional morphology during comprehension (9). In combination with the findings of Sahin et al., this suggests that Broca's area is recruited during both language production and comprehension. Whether these recruitments can be separated at the scale of the microcircuitry within Broca's area remains to be seen.

Broca's area has been proposed to have a more specialized role in language processing - facilitating linguistically motivated operations of syntactic movement (10) and processing hierarchical structures (11). The 
results of Sahin et al. indicate that the role of Broca's area is not so limited, but should be characterized in more general terms. It is likely involved in unification operations at the word and sentence level, in connection with temporal regions that are crucial for memory retrieval (12).

As is known for neurons in the visual cortex (13), the specific contribution of Broca's area may well vary with time, as a consequence of the different dynamic cortical networks in which it is embedded at different time slices. This fits well with the finding that Broca's area is not language specific, but is also recruited in the service of other cognitive domains, such as music (14) and action (15), and with the finding that its contribution to language processing crosses the boundaries of semantics, syntax, and phonology.

\section{References}

1. W. J. M. Levelt, Speaking: From Intention to Articulation (MIT Press, Cambridge, MA, 1989).

2. W. J. M. Levelt, Proc. Natl. Acad. Sci. U.S.A. 98, 13464 (2001).

3. W. J. M. Levelt, A. Roelofs, A. S. Meyer, Behav. Brain Sci. 22, 1 (1999).

4. G. S. Dell, M. F. Schwartz, N. Martin, E. M. Saffran, D. A. Gagnon, Psychol. Rev. 104, 801 (1997).

5. P. Broca, Bull. Soc. Anthropol. Paris 6, 377 (1865).
6. N. T. Sahin, S. Pinker, S. S. Cash, D. Schomer, E. Halgren, Science 326, 445 (2009).

7. P. Hagoort, M. van Turennout, in Speech Motor Production and Fluency Disorders: Brain Research in Speech Production, W. Hulstijn, H. Peters, P. Van Lieshout, Eds. (Elsevier, Amsterdam, 1997), pp. 351-361.

8. P. Indefrey, W. J. M. Levelt, Cognition 92, 101 (2004).

9. L. K. Tyler, W. Marslen-Wilson, Philos. Trans. R. Soc. 363, 1037 (2008).

10. Y. Grodzinsky, A. Santi, Trends Cogn. Sci. 12, 474 (2008).

11. A. D. Friederici, Trends Cogn. Sci. 8, 245 (2004).

12. P. Hagoort, Trends Cogn. Sci. 9, 416 (2005)

13. V. A. Lamme, P. R. Roelfsema, Trends Neurosci. 23, 571 (2000)

14. A. D. Patel, Nat. Neurosci. 6, 674 (2003).

15. F. Hamzei et al., Neuroimage 19, 637 (2003).

10.1126/science.1181675
PALEONTOLOGY

\section{Becoming T. rex}

James Clark

G igantic, ferocious, long-dead animals like Tyrannosaurus rex never fail to capture people's attention, and the discovery of a new tyrannosaurgiant or otherwise-is always big news. On page 418 of this issue, Sereno et al. (1) report on a spectacular skeleton of a new genus and species near the ancestry of the group including T. rex and its closest relatives, the Tyrannosauridae. At an estimated $3 \mathrm{~m}$ total length, Raptorex kriegsteini is much smaller than the largest $T$. rex [12.8 $\mathrm{m}$ long (2)] and other tyrannosaurids, but has several key features previously known only in this family. Raptorex thus provides a glimpse at how tyrannosaurids evolved.

Department of Biological Sciences, George Washington University, Washington, DC 20052, USA. E-mail: jclark@ gwu.edu
Fossils preserved in the rock with Raptorex point strongly to its origin from the beds at the bottom of the Jehol Group in northeastern China, although the locality remains unknown. The Jehol Group fossil beds (3) are famous for preserving dinosaurs with feathers in their thin-bedded shales, including the basal tyrannosaur Dilong (4), but the skeletons are usually crushed into two dimensions, and structures such as the skull are difficult to study. Fortunately, a series of beds in the lowest part of the Jehol Group yields exquisitely preserved, uncrushed skeletons, albeit without any soft tissues.

The Raptorex specimen was purchasedafew years ago by Henry J. Kriegstein at the Tucson Gem and Mineral Show (5), a venue notorious for the sale of illegally collected fossils, such as the famous Archaeoraptor chimera from the Jehol Group (6). Kriegstein approached Sereno
A small tyrannosaur from the Early Cretaceous sheds light on the origin of predatory features of Tyrannosaurus rex.

with the fossil, and Sereno agreed to describe it on the condition that it would be deposited in a collection in China (5). Although the fossil is currently with Sereno in Chicago, the specimen will be deposited in the Long Hao Institute of Geology and Paleontology in Hohhot, Inner Mongolia. Lin Tan of that institute is a coauthor of the paper, along with Kriegstein.

What to do with "hot" specimens is a conundrum for scientists. Such specimens almost always lack reliable locality data and therefore information about the sediments in which they were preserved. Stolen fossils can preserve data about the anatomy of a new or poorly known species, but enriching thieves or their fences is no more ethical for a fossil than for a car or a Grecian statue. The naming of a new ankylosaur, Minotaurasaurus ramachandrani (7), was strongly criticized (8), because the fossil was almost certainly
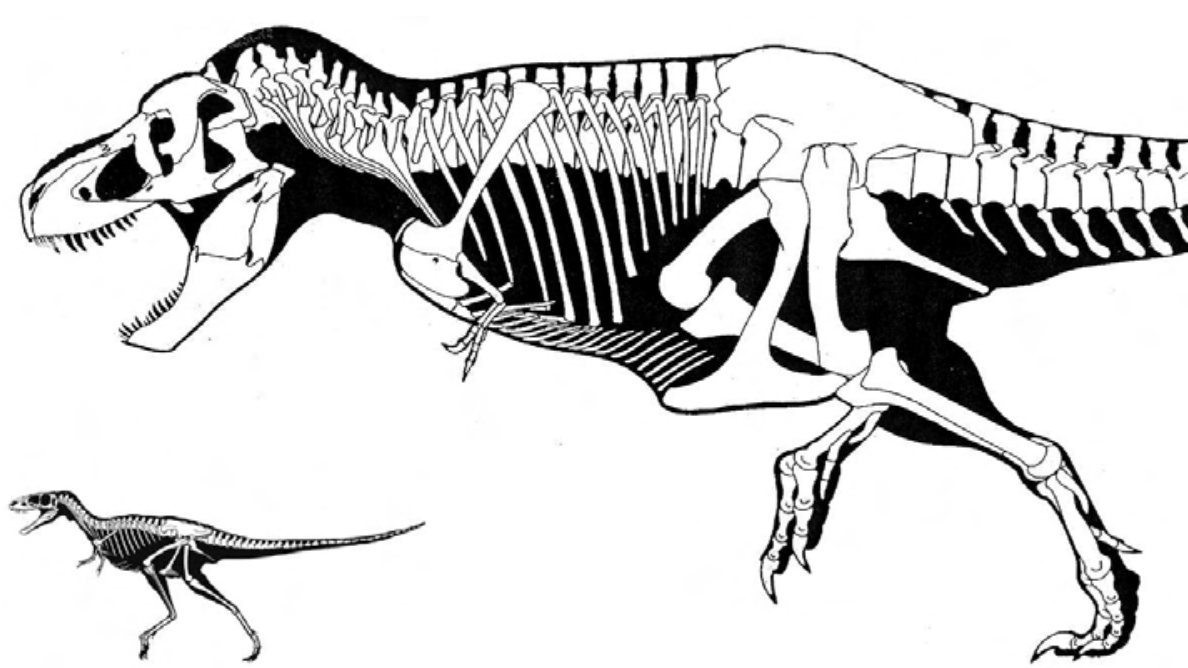

Raptorex kriegsteini (bottom left) (1) is dwarfed by the skeleton of Tyrannosaurus rex. 